\title{
Signaling Pathways of the TREM-1- and TLR4-Mediated Neutrophil Oxidative Burst
}

\author{
Philipp Haselmayer ${ }^{\mathrm{a}} \quad$ Martin Daniel $^{\mathrm{a}}$ Christine Tertilt $^{\mathrm{a}} \quad$ Helmut R. Salih $^{\mathrm{c}}$ \\ Michael Stassen ${ }^{a}$ Hansjörg Schild ${ }^{a}$ Markus P. Radsak ${ }^{a, b}$ \\ ${ }^{\mathrm{a}}$ Institute for Immunology, and ${ }^{\mathrm{b}}$ 3rd Department of Medicine, Johannes Gutenberg University, Mainz, \\ 'Department of Hematology/Oncology, Eberhad Karls University, Tübingen, Germany
}

\section{Key Words}

Polymorphonuclear neutrophils $\cdot$ Signal transduction •

TREM-1 $\cdot$ TLR4 $\cdot$ Oxidative burst $\cdot$ PI3-K $\cdot$ P38MAPK

\begin{abstract}
The triggering receptor expressed on myeloid cells 1 (TREM1) is involved in the innate inflammatory response to microbial infections. Activation and expression of TREM-1 by polymorphonuclear neutrophils (PMN) occurs in concert with Toll-like receptors (TLR) such as TLR4 for bacterial lipopolysaccharide. However, it is currently unclear how this is mediated on a molecular level. Using pharmacological inhibitors and Western blot analysis we demonstrate that phosphatidyl inositide 3-kinase, phospholipase $C$ and the mitogenactivated kinase P38MAPK are essential for the TREM-1- and TLR4-induced oxidative burst of human PMN. The activation of protein kinase B and extracellular signal-related kinase show characteristic phosphorylation patterns upon single or co-ligation indicating individual activation pathways of both receptors. Taken together, we provide new insights into the mechanisms how TREM- 1 and TLR interact creating synergistic activation in PMN. These results shed a new light on our understanding of how the innate inflammatory responses are regulated and might contribute to the development of future concepts for the treatment of severe inflammatory conditions such as sepsis.
\end{abstract}

Copyright $\odot 2009$ S. Karger AG, Basel (c) 2009 S. Karger AG, Basel

1662-811X/09/0016-0582\$26.00/0

Fax +41613061234 E-Mail karger@karger.ch www.karger.com
Accessible online at:

www.karger.com/jin

\section{Introduction}

Polymorphonuclear neutrophils (PMN) take major part in the innate host defense against pathogenic microorganisms like fungi and bacteria $[1,2]$. They recognize conserved microbial structures, so-called pathogenassociated molecular patterns, directly via cell surface receptors (that is, Toll-like receptors, TLR) [3-5] or cytoplasmic receptors such as NAIP, CIITA, HET-E, TP-1leucine-rich repeat (NACHT-LRR; NLR) [6, 7]. TLRmediated activation in PMN is dependent on the myeloid differentiation factor 88 (MyD88) [8] that induces downstream activation of phosphatidyl inositide 3-kinase (PI3K) [9] and mitogen-activated (MAP) kinases, including $\mathrm{p} 38 \mathrm{MAPK}$, extracellular signal-related kinase (ERK) and Jun $\mathrm{N}$-terminal kinase (JNK) $[8,10]$, ultimately leading to the activation of the transcription factor NF- $\mathrm{KB}$ that regulates the transcription of target genes [11].

The triggering receptor expressed on myeloid cells (TREM)- 1 is a $30-\mathrm{kDa}$ transmembrane receptor of the immunoglobulin superfamily constitutively expressed on PMN and monocytes.

In PMN, ligation of TREM-1 activates the full spectrum of neutrophil effector mechanisms, including the release of inflammatory mediators such as IL-8, MCP-1 or myeloperoxidase, degranulation, phagocytosis and the oxidative burst in synergy with TLR agonists [12-14]. Beyond this, TREM-1 has been recognized as an impor-

Dr. Markus P. Radsak

Institute for Immunology, University of Mainz

Obere Zahlbacher Strasse 67

DE-55131 Mainz (Germany)

Tel. +496131 393 7156, Fax +49 6131393 6688, E-Mail radsak@uni-mainz.de 
tant regulator of innate inflammatory responses. This is illustrated by rodent models where blocking of TREM-1mediated signals is protective in endotoxic shock, peritonitis or pneumonia [15-17]. Although the natural ligands for TREM-1 have not yet been identified, some authors describe the occurrence of a TREM-1 ligand in the serum of some septic patients $[18,19]$. In addition, filoviruses can directly activate cells via TREM-1, implying the presence of viral TREM-1 ligands [20]. However, the fact that TREM-1 is of importance in the course of endotoxic shock strongly argues for a so far unidentified endogenous ligand that we recently located on platelets [14].

For activation, TREM-1 needs to associate with the adaptor molecule DAP12 [12, 21]. Concurrent ligation of TLRs [12, 13, 15, 22] or NLRs [23] synergistically enhances the TREM-1-mediated activation of PMN and monocytes. TREM-1 ligation with specific antibodies leads to the activation of phospholipase C (PLC), $\mathrm{Ca}^{2+}$ mobilization as well as activation of the MAP kinases ERK and p38MAPK, important prerequisites for the initiation of neutrophil effector functions $[12,24]$. Beyond this, the activation of the src kinase Lyn, PI3K and the JAK-STAT pathway after TREM-1 ligation has been described [25]. For the synergistic action, the recruitment of TREM-1 and TLR4 together in GM1 ganglioside lipid rafts seems to be relevant [25]. To this end, a PI3K-dependent pathway is important for the synergistic activation of TREM1 and LPS in monocytes [26]. Nevertheless, whether this mechanism is also relevant in PMN has not been addressed so far.

Therefore, we analyzed the signaling events induced by ligation of TREM-1 and TLR4 in human PMN investigating the functional impact of pharmacological inhibitors of key signaling events in the induction of the oxidative burst. Additional studies by Western blot identified PI3K and p38MAPK activation as essential events for both LPS- and TREM-1-mediated activation that is initiated independent of each other. Taken together, we map key signaling pathways involved in TREM-1- and TLR4mediated activation and disclose two distinct signaling cascades that connect TREM-1 with TLR4 signaling in PMN. Our results provide an enhanced understanding of PMN-mediated inflammatory responses and might contribute to the development of future concepts for the treatment of severe inflammatory responses like sepsis.

\section{Materials and Methods}

\section{Materials}

LPS from Salmonella typhimurium, NaF, Na-pyrophosphate, Na-orthovanadate, PMSF and aprotinin were from Sigma-Aldrich (Taufkirchen, Germany). NP40, Triton X-100, sodium dodecyl sulfate, Tris and $\mathrm{NaCl}$ were from Carl Roth (Karlsruhe, Germany). Leupeptin and Pepstatin were from Peptanova (Sandhausen, Germany). Antibodies against phospho-ERK1/2, total ERK1/2, phospho-p38MAPK, total p38MAPK, phospho-PKB and total PKB were purchased from Cell Signaling (Danvers, Mass., USA). The monoclonal antibodies (mAbs) anti-TREM-1 (clone 1C5) and monoclonal mouse IgG1 clone 4C9 used as isotype control were purified from hybridoma supernatants as described previously [14]. All mAbs used for activation were tested endotoxin-free $(<0.1 \mathrm{EU} / \mu \mathrm{g}$ protein) determined by a limulus amoebocyte lysate assay (QCL-1000; BioWhittaker, Verviers, Belgium). The PI3K inhibitor LY294002, PLC inhibitor U73122, p38MAPK inhibitor SB203580, the ERK inhibitor PD98059 and the PKB inhibitor triciribine were all purchased from Calbiochem (Darmstadt, Germany). For analyses by flow cytometry, FITClabeled anti-CD66b (Coulter Immunotech, Hamburg, Germany), PE-labeled anti-CD11b (BD Pharmingen, Franklin Lakes, N.J., USA), APC-labeled anti-TLR4 (eBioscience, San Diego, Calif., USA) were used. All human studies were performed after obtaining informed consent from healthy volunteer donors and were approved by the local ethics committee according to the institutional guidelines.

\section{PMN Purification}

PMN were separated from human blood using Polymorphprep (Axis-Shield, Oslo, Norway) as described previously [27]. Briefly, citrated whole blood was layered over the gradient medium in a $1: 1$ ratio and centrifuged at $450 \mathrm{~g}$ for $35 \mathrm{~min}$ at room temperature. After centrifugation, the lower PMN-containing band was harvested and washed with phosphate-buffered saline (Invitrogen, Karlsruhe, Germany). Contaminating red blood cells were removed by a hypotonic lysis step $[150 \mathrm{~mm}$ ammonium chloride, $1 \mathrm{~mm}$ potassium bicarbonate, $0.1 \mathrm{~mm}$ ethylene diamine tetra acetate (all from Sigma) in distilled water, $\mathrm{pH}$ 7.3]. Purity of cell preparation was assessed by flow cytometry with CD66b as marker for PMN. In general, 98\% of cells were CD66b-positive PMN with less than $0.2 \%$ monocytes assessed by CD14 expression via flow cytometry using a FACScanto flow cytometer and FACSDiva software (Becton Dickinson, Heidelberg, Germany). Alternatively, PMN were purified using CD15-coupled magnetic beads (Miltenyi, Bergisch-Gladbach, Germany) according to the manufacturer's instructions.

\section{Cell Stimulation}

Cross-linking of anti-TREM-1 was performed with a secondary goat anti-mouse $\mathrm{F}(\mathrm{ab})_{2}$ (Dianova, Hamburg, Germany) for experiments followed by $\mathrm{Ca}^{2+}$ mobilization assay or Western blot analyses. For assessment of the oxidative burst activity, antiTREM-1 was coated in 96-well flat-bottom plates (Greiner Bio One, Frickenhausen, Germany). Inhibitors were added $30 \mathrm{~min}$ before stimulation of cells. The oxidative burst induced by PMA (10 nM; Sigma-Aldrich) in the presence or absence of the respective inhibitors was used as viability control and had no impact on the PMA-induced oxidative burst at the concentrations used for 
TREM-1- or LPS-induced activation (online suppl. fig. S2, www. karger.com/doi/10.1159/000231973).

Detection of Oxidative Burst

The presence of hydrogen peroxide was detected by oxidation of nonfluorescent dichloro-fluorescein diacetate (Sigma-Aldrich) into green fluorescent dichloro-fluorescein as described previously [14]. Specific fluorescence index of stimulated cells was obtained by subtraction of the background fluorescence of labeled cells incubated in medium alone at the corresponding time points.

\section{Western Blot Analysis}

Cells were lysed with modified RIPA buffer [1\% NP40, 1\% Triton X, 0.1\% SDS, $0.01 \mathrm{M}$ Tris, $0.05 \mathrm{M} \mathrm{NaCl}, 0.05 \mathrm{M} \mathrm{NaF}, 0.005 \mathrm{M}$ Na-pyrophosphate, $0.5 \mathrm{mM} \mathrm{Na}$-orthovanadate, aprotinin, $0.2 \mathrm{mM}$ leupeptin, $0.1 \mathrm{~mm}$ pepstatin, $2 \mathrm{mM}$ PMSF and complete protease inhibitor cocktail], protein concentration was determined according to the method of Bradford. Samples were then subjected to $10 \%$ SDS PAGE. After electrophoresis, proteins were transferred from the gel onto a nitrocellulose membrane (Amersham, Piscataway, N.J., USA). The membranes were probed with appropriate primary and secondary antibodies conjugated with horseradish peroxidase as indicated and visualized by the ECL detection system as directed by the manufacturer (Pierce, Bonn, Germany). Quantitative signal density analyses were done by Quantity One software V 4.4.0 and given as average density in arbitrary units (BioRad, Munich, Germany).

Calcium Mobilization Assay

PMN $\left(2 \times 10^{6} / \mathrm{ml}\right)$ were loaded with the $\mathrm{Ca}^{2+}$-sensitive fluorogenic dye FLUO-3/AM ( $2 \mu \mathrm{M}$; Molecular Probes, Eugene, Oreg., USA) at $30^{\circ} \mathrm{C}$ for $30 \mathrm{~min}$ in medium and washed twice. Stimuli were administered until measurement in FACScanto analyzer (Becton Dickinson) and analyzed with FlowJo software V8.7.1 (Tree Star Inc., Ashland, Oreg., USA).

\section{Results}

\section{PI3K, PLC and $\mathrm{p} 38 \mathrm{MAPK}$ Are Essential for}

TREM-1- and LPS-Mediated Oxidative Burst

TREM-1 needs to associate with the adaptor molecule DAP12 to mediate the activation of p38MAPK and ERK along with the phosphorylation of PLC $[12,24]$. The synergy of TREM-1 with TLR ligands observed by others and us is an immediate effect (fig. 1a) and therefore we hypothesized that it is unlikely due to an altered surface expression of the receptors involved.

Accordingly, we were unable to detect an alteration of TREM-1 (not shown) or TLR4 surface expression (online suppl. fig. S1) upon stimulation up to $3 \mathrm{~h}$ excluding regulation of TREM-1 or TLR by degranulation. Instead, we assumed that this synergy is mediated by the amplification of intracellular signaling events that have not been characterized so far in PMN $[28,29]$. Thus, we assessed the oxidative burst activity induced upon TREM-1 and TLR4 ligation in the presence or absence of specific pharmacological inhibitors of signal transduction. For all inhibitor studies, we performed careful titration experiments and regarded only those inhibitor concentrations as nontoxic where the oxidative burst activity induced by phorbol ester (PMA) was unaffected (online suppl. fig. S2). As shown in figure la, we observed the induction of a weak but consistent oxidative burst activity in human PMN after ligation of TREM-1 using a monoclonal antibody and a stronger response after ligation of TLR4 by LPS. Co-ligation of both receptors resulted in a greatly enhanced oxidative burst activity. As shown in figure $1 \mathrm{~b}$ and c, the TLR4- as well as TREM-1-mediated oxidative burst was completely abrogated in the presence of the PI3K inhibitor LY294002 or the PLC inhibitor U73122, respectively, while the PMA-induced burst remained unaffected (online suppl. fig. S2). The p38MAPK inhibitor SB203580 also suppressed the TLR4- and TREM-1-mediated oxidative burst upon single receptor ligation leaving some minor residual burst activity after co-ligation of TREM-1 and TLR4 (fig. 1d).

Again, this inhibitor had no effect on the PMA-induced oxidative burst at the amount used (online suppl. fig. S2). In contrast, the MEK1 inhibitor PD98059 (fig. 1e) or the PKB inhibitor triciribine (fig. 1f) did not affect the TREM-1- or TLR4-induced oxidative burst, indicating no role for ERK or PKB for this particular effector function. Thus, this set of data indicates an essential role for PI3K, PLC and p38MAPK in the TREM-1 as well as the TLR4 activation pathway for the induction of the oxidative burst.

\section{Biphasic Activation of $P K B$ and MAP Kinases \\ ERK and p38MAPK upon TREM-1-/LPS-Mediated Activation}

To further study TREM-1-/TLR4-mediated activation of PMN on a biochemical level, we performed Western blot analyses for phosphorylation of $\mathrm{PKB}$ as a direct downstream event of PI3K, for the activation of ERK and p38MAPK which both are involved in the activation pathways of TREM-1 and TLR4 $[12,24,26]$. In a time course analysis after ligation of TREM-1 shown in figure $2 \mathrm{a}$, we detected phosphorylated PKB at early time points at 1-3 min that decayed rapidly afterwards. In contrast, PKB phosphorylation upon TLR4 stimulation occurred clearly at a later time with a maximum at 30 min. Interestingly, upon co-ligation of both receptors, we observed a biphasic activation pattern for PKB: first at an early time point corresponding with the phosphorylation 


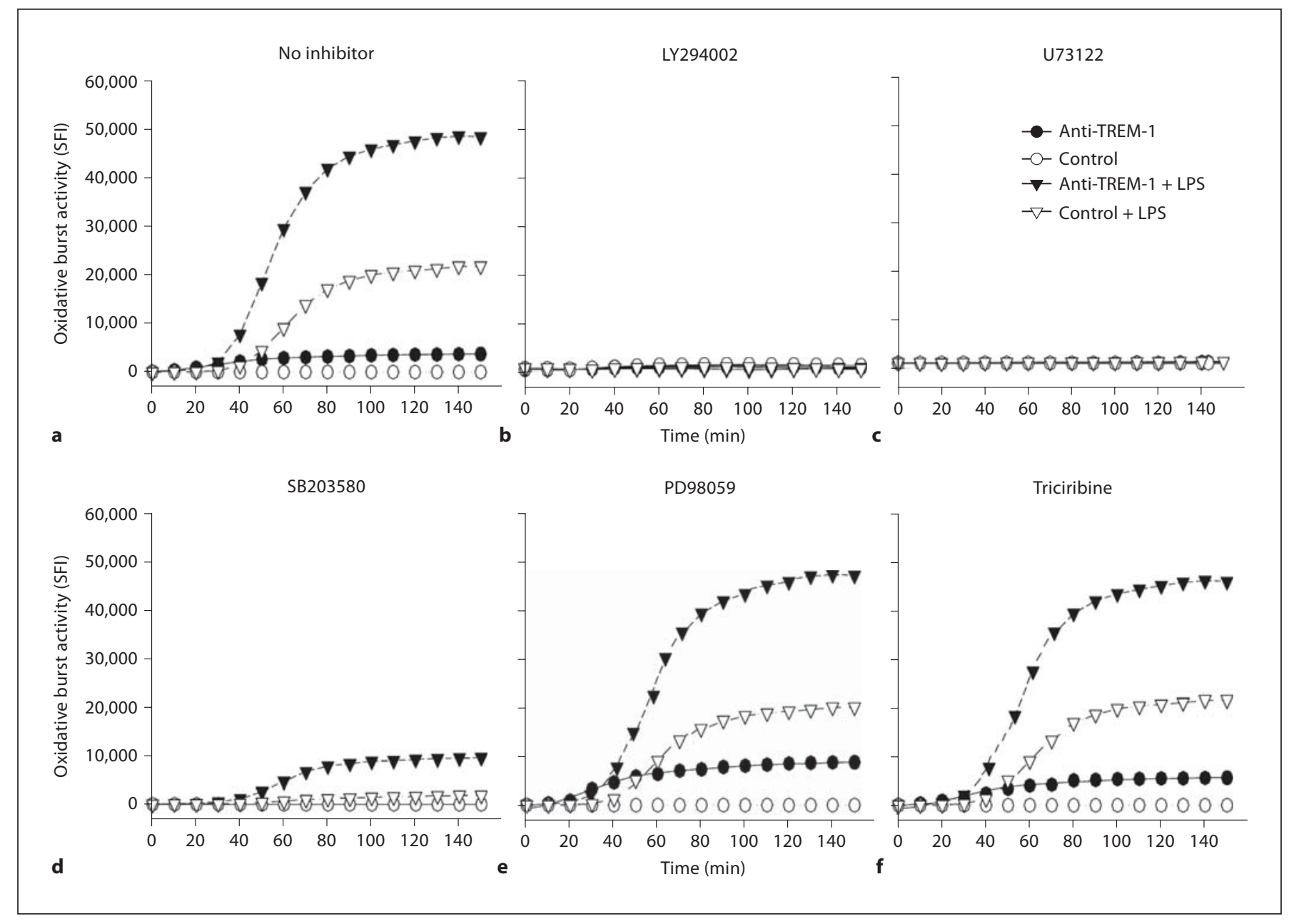

Fig. 1. PI3K, PLC and p38MAPK are essential for the TREM-1and TLR4-induced oxidative burst. The oxidative burst activity of human PMN $\left(2 \times 10^{5}\right.$ per well $)$ was assessed upon stimulation with anti-TREM-1 $(10 \mu \mathrm{g} / \mathrm{ml})$, control $\mathrm{mAb}$ (control, $10 \mu \mathrm{g} / \mathrm{ml})$, control with LPS (100 ng/ml) or anti-TREM-1 with LPS. The $\mathrm{x}$ axis indicates time (min) after ligation; the $y$-axis indicates the oxidative burst activity as specific fluorescence index (SFI) calculated as described in Materials and Methods. a Oxidative burst in absence of an inhibitor (no inhibitor). b PMN were pre-incubated with the PI3 kinase inhibitor LY294002 (5 $\mu \mathrm{M})$ for $30 \mathrm{~min}$ at $37^{\circ} \mathrm{C}$ before stimulation as in a. c PMN were pre-incubated with the PLC inhibitor U73122 (0.3 $\mu \mathrm{M})$ for $30 \mathrm{~min}$ at $37^{\circ} \mathrm{C}$ before stimulation as in a.d PMN were pre-incubated with the $338 \mathrm{MAPK}$ inhibitor SB203580 (20 nM) for $30 \mathrm{~min}$ at $37^{\circ} \mathrm{C}$ before stimulation as in a. e PMN were pre-incubated with the pERK inhibitor PD98059 $(20 \mu \mathrm{M})$ for $30 \mathrm{~min}$ at $37^{\circ} \mathrm{C}$ before stimulation as in $\mathbf{a}$. f $\mathrm{PMN}$ were pre-incubated with the $\mathrm{PKB}$ inhibitor triciribine $(20$ $\mu \mathrm{M})$ for $30 \mathrm{~min}$ at $37^{\circ} \mathrm{C}$ before stimulation as in a. As viability control, the oxidative burst activity induced by PMA was not impaired in the presence of the inhibitors at the concentration used (online suppl. fig. S2). The results from 1 representative of at least 3 independent experiments with different healthy donors are depicted as the mean with standard deviation from triplicate wells. pattern of TREM-1 ligation alone, and second at a later time point parallel to the TLR4-induced phosphorylation of PKB. For the phosphorylation of ERK upon TREM-1 or TLR4 ligation we observed an activation pattern resembling very much the one described for PKB (fig. 2b), again with an early TREM-1 induced phosphorylation signal at $3 \mathrm{~min}$, and a late TLR4-mediated signal after
30-60 min of stimulation. Co-ligation led once again to an overlap of both phosphorylation signals at 3 and 30 min, suggesting that PKB and ERK phosphorylation might be linked together.

On the contrary, performing a kinetic analysis for p38MAPK activation (fig. 2c), we found a slightly later increase in p38MAPK phosphorylation starting at $10 \mathrm{~min}$ 


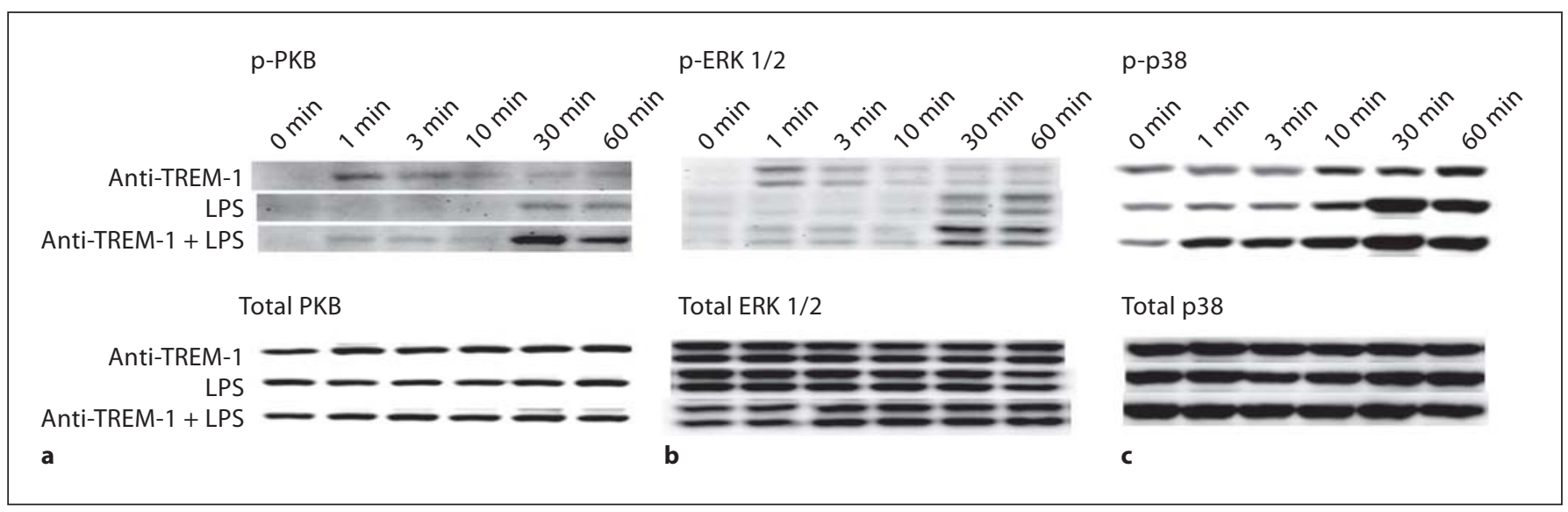

Fig. 2. Ligation of TREM-1 and TLR4 induces a distinct phosphorylation pattern for PKB, ERK and p38MAPK. PMN $(2 \times$ $\left.10^{6} / \mathrm{ml}\right)$ were stimulated with anti-TREM- $1[10 \mu \mathrm{g} / \mathrm{ml}$ and crosslink with goat anti-mouse $\mathrm{F}(\mathrm{ab})_{2}, 20 \mu \mathrm{g} / \mathrm{ml}$, LPS $(100 \mathrm{ng} / \mathrm{ml})$ or both for the indicated time periods from 0 to $60 \mathrm{~min}$. Whole cell lysates were prepared and separated by $10 \%$ SDS-PAGE and subsequently analyzed by Western blot against phosphorylated PKB (a), phosphorylated ERK1/2 (b) and phosphorylated p38MAPK

after TREM-1 or TLR4 ligation with a maximum after 30 min. Co-ligation of TREM-1 and TLR4 induced a longlasting $\mathrm{p} 38 \mathrm{MAPK}$ activation starting at $1 \mathrm{~min}$ and lasting until $60 \mathrm{~min}$, suggestive of a synergistic activation and a signaling pathway different from PKB and ERK activation.

An Important Role for PI3K in TREM-1- and TLR4Induced Activation of $P K B, E R K$ and $P 38 M A P K$

To assess whether the activation of PKB, ERK and p38MAPK are connected to PI3K, we analyzed the phosphorylation of these molecules in the presence of the PI3K inhibitor LY294002 at early (3 min) or late time points (30 min) after ligation of TREM-1, TLR4 or co-ligation. As shown in figure 3 , we found that the activation of $\mathrm{PKB}$ (fig. 3a) and ERK (fig. 3b) were completely abrogated at the early as well as late time points for single and co-ligation of TREM-1 and TLR4 in the presence of PI3K inhibition. This indicates that the phosphorylation of PKB and ERK both occur downstream of PI3K for TREM-1- and TLR4mediated signals. Moreover, for co-ligation of both receptors PI3K is still required, suggesting that TREM-1- as well as TLR4-induced signals adhere to their signaling pathways. As furthermore shown in figure $3 c$, the presence of the PI3K inhibitor did not affect the enhanced TREM-1and TLR4-induced phosphorylation of p38MAPK at 30 (c). The respective protein loading controls blotted against total PKB, total ERK1/2 and total p38MAPK showing no differences among the differently activated cells are shown in the lower panels. In online supplement table S1, the corresponding densitometry analyses of the depicted blots are shown as the average density (arbitrary units). The depicted results are representative of 3 independent experiments. min, suggesting that PI3K is not involved in the TLR4- or TREM-1-induced activation of p38MAPK. Correspondingly, we were unable to detect a relevant difference in TLR4-/TREM-1-induced phosphorylation of PKB in the presence of the p38MAPK inhibitor SB203580 (fig. 4a). Interestingly, upon TLR4/TREM-1 co-ligation there was a weaker phosphorylation of ERK in the presence of the p38MAPK inhibitor SB203580 indicating the activation of ERK occurs downstream of PI3K, but partially depends on p38MAPK (fig. 4b). Consistent with previous data [30], the inhibitor SB203580 does not inhibit phosphorylation of $\mathrm{p} 38 \mathrm{MAPK}$, but downstream activation. Therefore, the detection of phospho-p38MAPK in the presence of the specific inhibitor is expected (fig. 4c). Thus, a potential mechanism of synergy between these receptors may be mediated via the PI3K-independent activation of p38MAPK both targeting ERK.

\section{TREM-1- and TLR4-Induced $\mathrm{Ca}^{2+}$ Signals Are Dependent on PI3K and PLC}

We observed an essential role for PLC in the induction of the respiratory burst upon TREM-1 and TLR4 ligation (fig. 1c). PLC cleaves phosphatidyl inositol 4,5-bisphosphate into inositol-1,4,5-trisphosphate which triggers an increase in intracellular $\mathrm{Ca}^{2+}$, and 1,2-diacylglycerol that in turn activates protein kinase $\mathrm{C}$; the latter is a crucial 
Fig. 3. PI3K has an essential role for the TREM-1- and TLR4-mediated activation of PKB and ERK, but not for p38MAPK. PMN $\left(2 \times 10^{6} / \mathrm{ml}\right)$ were stimulated as described in figure 2 and analyzed for the phosphorylation of PKB (a), ERK1/2 (b) and p38MAPK (c) in the absence or presence of the PI3K inhibitor LY294002 ( $5 \mu \mathrm{M}$; added $30 \mathrm{~min}$ before stimulation) after stimulation for the indicated time periods. The respective protein loading controls in the presence or absence ofLY294002 $(5 \mu \mathrm{M})$ blotted against total $\mathrm{PKB}$, total ERK1/2 and total p38MAPK showed no differences among the differently activated cells (lower panels). In online supplement table S2, the corresponding densitometry analyses of the depicted blots are shown as the average density (arbitrary units). These results are representative of 3 independent experiments with different healthy donors.

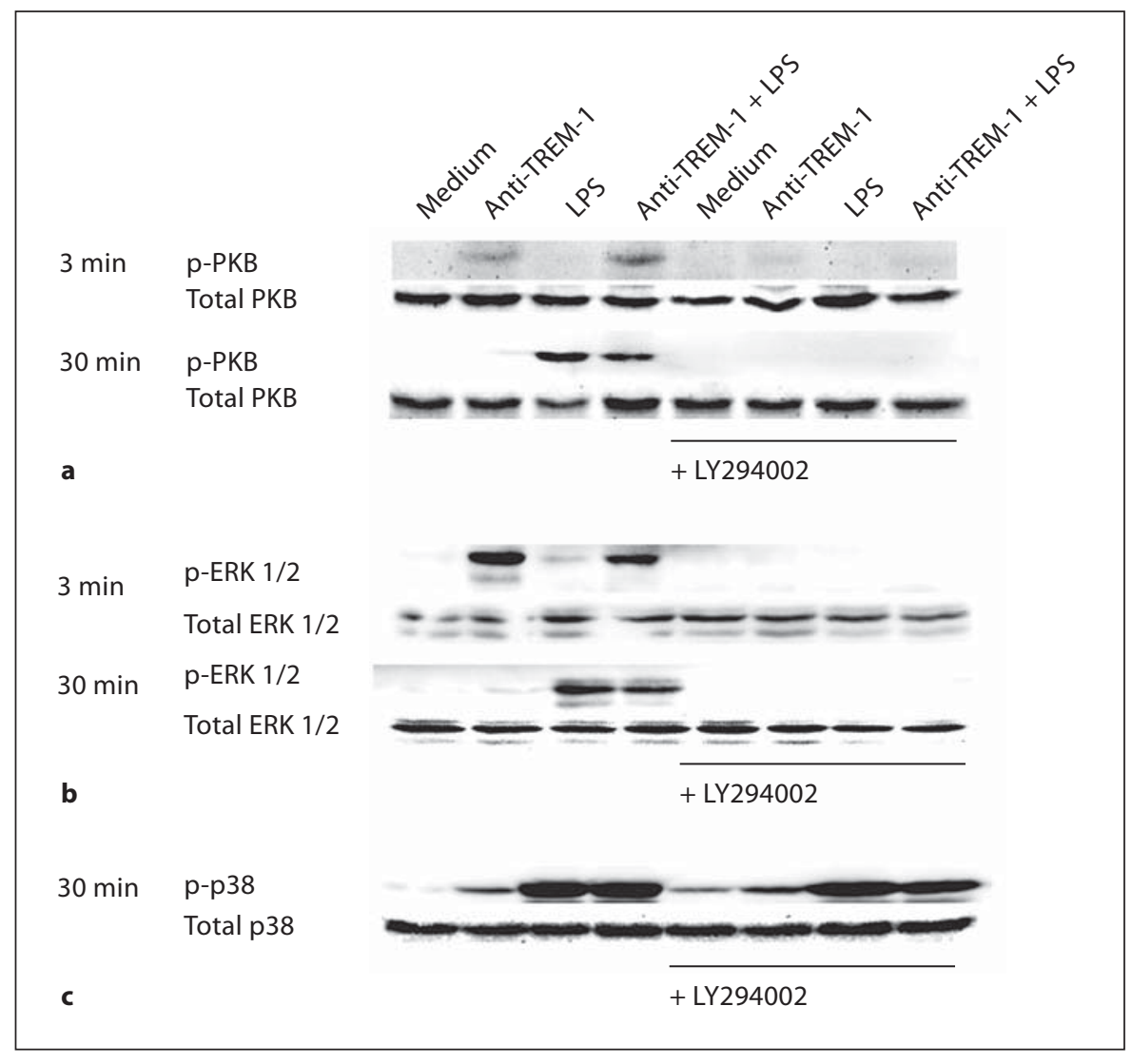

step for the initiation of the oxidative burst in PMN [31, 32]. Hence, we analyzed intracellular $\mathrm{Ca}^{2+}$ levels in PMN loaded with the $\mathrm{Ca}^{2+}$-sensitive fluorogenic dye FLUO3AM upon TREM-1, TLR4 ligation or co-ligation of both. We found that ligation of TREM-1 induced a transient increase in intracellular $\mathrm{Ca}^{2+}$ levels as described previously (fig. 5a, grey line) [12]. In contrast to this and in line with previous data [33], we were unable to observe $\mathrm{Ca}^{2+}$ flow induced by TLR4 ligation alone (data not shown). However, TREM-1 and TLR4 co-ligation resulted in a marked increase in intracellular $\mathrm{Ca}^{2+}$ levels (fig. 5a, black line), suggesting that TREM-1- and TLR4-derived signaling cascades converge to an increased $\mathrm{Ca}^{2+}$ signal. Since the inhibition of PI3K and PLC completely abrogated the oxidative burst, we were interested whether the intracellular $\mathrm{Ca}^{2+}$ flow was influenced by these inhibitors. Therefore, we assessed the intracellular $\mathrm{Ca}^{2+}$ levels upon single TREM-1 ligation and co-ligation with TLR4 in the presence of the PI3K inhibitor LY294002 (fig. 5) or the PLC inhibitor U73122 (fig. 5). In both cases, we were unable to detect any significant $\mathrm{Ca}^{2+}$ signal, suggesting that the activation of PLC and subsequent $\mathrm{Ca}^{2+}$ flow mediated by the upstream activation of PI3K are crucial for the initiation of the respiratory burst in PMN. In contrast, the p38MAPK inhibitor SB203580 had no influence on the $\mathrm{Ca}^{2+}$ flow induced by TREM-1 and TLR4 (fig. 5), suggesting that the p38MAPK-dependent oxidative burst activity does not need $\mathrm{Ca}^{2+}$ signals and is involved in an activation pathway different from the activation of PI3K and PLC.

\section{Discussion}

TREM-1 contributes to the amplification of the innate inflammatory response in severe infections and sepsis $[15,21]$. Recent findings by us suggest that the so far unknown ligand for TREM-1 is present on platelets and enhances the LPS-induced activation of PMN [14]. However, it has been so far unclear how TLR- and TREM-1induced signals are connected within PMN. The only report addressing the mechanisms of synergy demonstrates that TREM-1 and TLR4 need to co-localize in lipid rafts [25]. While TLR4 signaling in human PMN is 
Fig. 4. TREM-1- and TLR4-mediated activation of $\mathrm{PKB}$ and ERK is independent of p38MAPK. PMN $\left(2 \times 10^{6} / \mathrm{ml}\right)$ were stimulated as described in figure 2 and analyzed for the phosphorylation of PKB (a), ERK1/2 (b) and p38MAPK (c) in the absence or presence of the $\mathrm{p} 38 \mathrm{MAPK}$ inhibitor SB203580 (20 nM; added $30 \mathrm{~min}$ before stimulation) after stimulation for the indicated time periods. The respective protein loading controls in the presence or absence of SB203580 (20 nM) blotted against total $\mathrm{PKB}$, total ERK1/2 and total p38MAPK showed no differences among the differently activated cells (lower panels). In online supplement table S3, the corresponding densitometry analyses of the depicted blots are shown as the average density (arbitrary units). These results are representative of 3 independent experiments with different healthy donors.

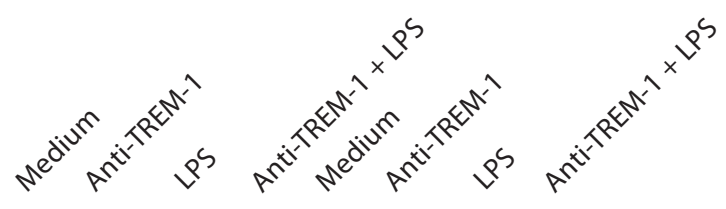

$3 \min \quad \mathrm{p}-\mathrm{PKB}$

Total PKB

30 min $\quad \mathrm{p}-\mathrm{PKB}$

Total PKB

a

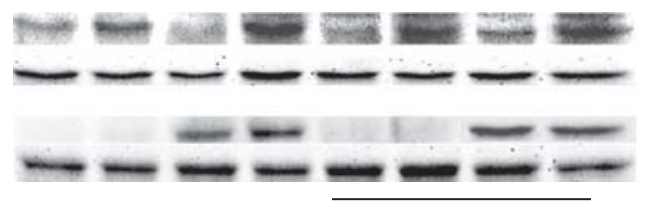

+ SB203580

$3 \min \quad$ p-ERK $1 / 2$

Total ERK $1 / 2$

p-ERK $1 / 2$

Total ERK $1 / 2$

b

+ SB203580

$30 \min \quad \mathrm{p}-\mathrm{p} 38$

Total p38

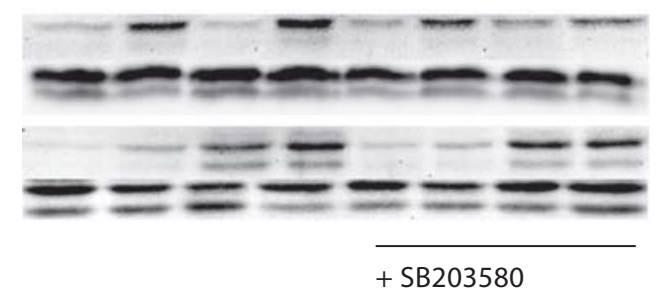

C

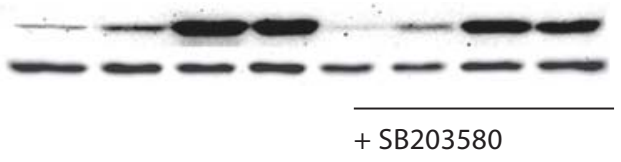

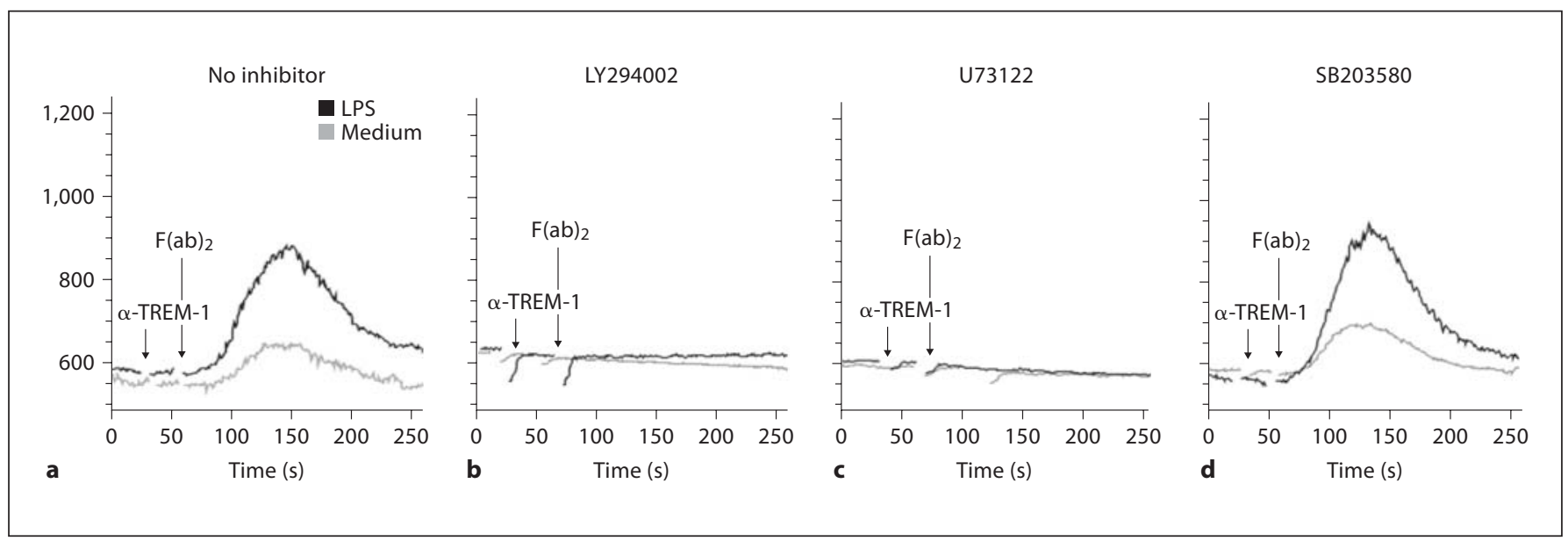

Fig. 5. TLR4 ligation enhances the TREM-1-induced $\mathrm{Ca}^{2+}$ flow in a PI3K- and PLC-dependent manner. PMN $\left(2 \times 10^{6}\right.$ cells $\left./ \mathrm{ml}\right)$ were loaded with the $\mathrm{Ca}^{2+}$-sensitive fluorogenic dye FLUO3-AM. Anti-TREM-1 $(10 \mu \mathrm{g} / \mathrm{ml})$ and goat anti-mouse $\mathrm{F}(\mathrm{ab})_{2}(20 \mu \mathrm{g} / \mathrm{ml})$ were added as indicated and $\mathrm{Ca}^{2+}$ flow was monitored as green fluorescence (y-axis) over time (x-axis) in the absence (grey lines) or presence (black lines) of LPS $(100 \mathrm{ng} / \mathrm{ml}) . \mathrm{Ca}^{2+}$ flow upon TREM-1 and/or TLR4 ligation by LPS is depicted in the absence of an inhibitor (a), in the presence of the PI3K inhibitor LY294002 ( $5 \mu \mathrm{M}$; added $30 \mathrm{~min}$ before stimulation; $\mathbf{b}$ ) or in the presence of the PLC inhibitor U73122 $(0.3 \mu \mathrm{M}$; added 30 min before stimulation; c) or the p38MAPK inhibitor SB203580 (20 $\mu \mathrm{M}$; added 30 min before stimulation; d). The results from 1 representative of 4 independent experiments with different healthy donors are depicted. 
Fig. 6. A proposed model of TREM-1- and TLR4-induced signal transduction for the induction of the PMN oxidative burst. TREM-1- and TLR4-mediated signaling pathways are initiated independently. TLR4 induces the PI3K-independent activation of $\mathrm{p} 38 \mathrm{MAPK}$ and the PI3K-dependent activation of PKB, MAPK ERK and PLC triggering TRP channels. TREM-1induced signaling is crucially dependent on PI3K leading to the activation of p38MAPK, ERK, PKB and PLC triggering Ca flow. The synergy between TREM-1 and TLR4 might occur via at least two distinct mechanisms: the TREM-1-induced PI3K-dependent and the TLR4-induced PI3K-independent activation of p38MAPK and via a PI3K-dependent distinct leading to an enhanced oxidative burst activity of PMN.

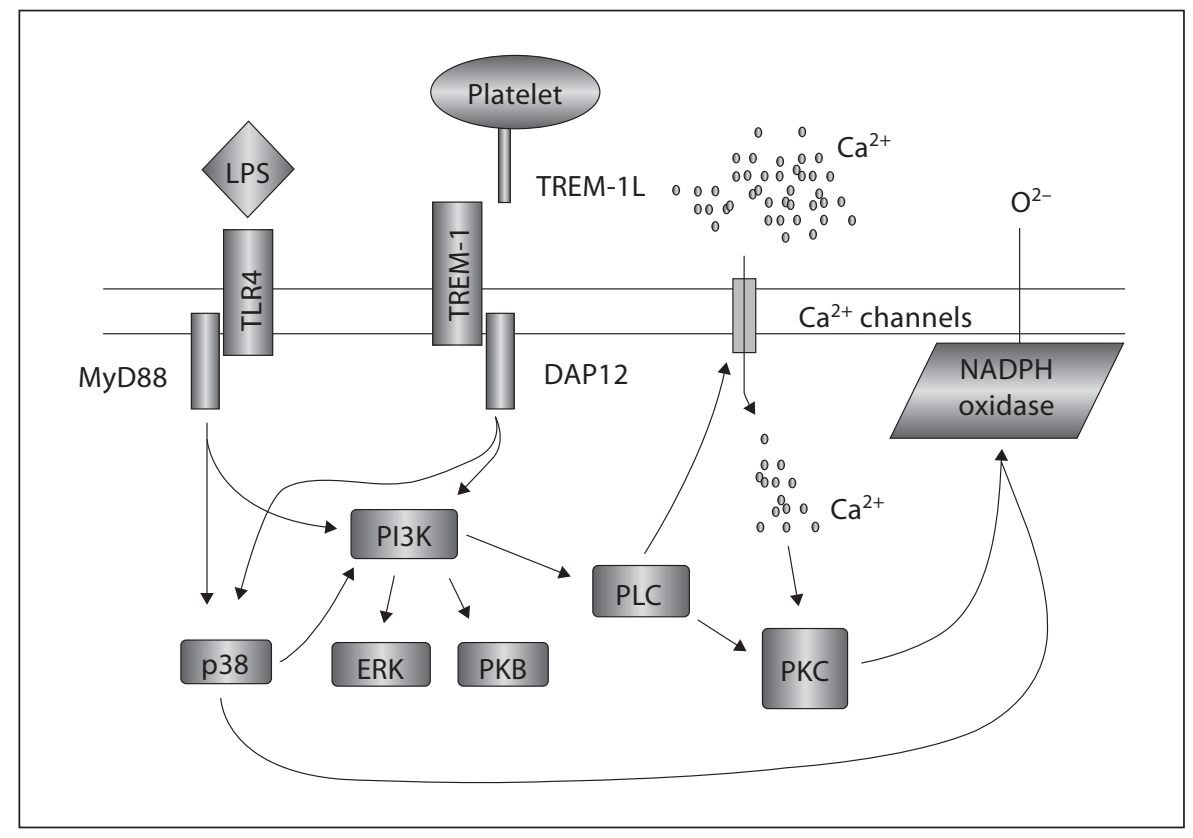

solely dependent on the adaptor protein MyD88 [8], TREM-1 needs to associate with DAP12 for activation $[12,15]$. Hence, distinct signaling pathways are engaged that need to intersect at some point to explain the functional synergy of these 2 receptors. As an important and immediate PMN effector function, we analyzed the oxidative burst activity upon TREM-1 and TLR4 ligation. Assembly and activation of the NADPH oxidase is crucially dependent on the activation of protein kinase $\mathrm{C}$ which occurs downstream of PLC [34]. Along this line, our results clearly demonstrate that the activation of PI3K and PLC are essential and represent a common pathway for induction of the TREM-1- and the TLR4-induced oxidative burst in PMN. The activation of PI3K is important for the induction of the neutrophil oxidative burst in general [35] and also essential for the burst activity induced by TLR4 and TREM-1 (fig. 1). Interestingly, we observed a biphasic phosphorylation of $\mathrm{PKB}$ as a direct downstream event of PI3K upon TLR4/TREM-1 co-ligation indicating that TLR4 and TREM-1 utilize distinct receptor proximal signaling pathways to activate PI3K that still remain to be determined in detail. While the TLR4 pathway is entirely dependent on MyD88 in PMN [8], for TREM-1 the src kinases like Syk and Lyn seem to be important $[21,26,29,36]$ although the exact role in PMN still needs to be determined.

The activation of ERK upon TREM-1 $[12,25]$ or TLR4 $[37,38]$ ligation has been described previously. We found that ERK also follows a biphasic phosphorylation pattern that is abrogated by inhibition of PI3K (fig. 3a and b) clearly placing the activation of ERK downstream of PI3K for TREM-1- as well as TLR4-induced signals. The p38MAPK is involved and essential in many biological processes [39] and also has a role in the MyD88-dependent signaling pathway after TLR4 [40] and upon TREM1 ligation [24]. We were here able to confirm these results and beyond this found an enhanced activation of p38MAPK after TREM-1/TLR4 co-ligation. In addition, p38MAPK is partially involved in the activation of ERK (fig. 4b), implying a crosstalk with the PI3K pathway. In line with others [34], our data indicate that p38MAPK activation is critical for initiation of the oxidative burst induced by TREM-1 and TLR4 by an activation pathway independent of PI3K and $\mathrm{Ca}^{2+}$ signaling. It appears likely that TLR4- and TREM-1-induced p38MAPK activation results in a direct activation of the NADPH oxidase as described for the TNF- $\alpha$ - or f-MLP-induced oxidative burst [41]. This suggests that one mechanism of synergy between TREM-1 and TLR4 involves the PI3K-independent activation of p38MAPK. Whether this signaling cascade in PMN is also present in monocytes like the PI3Kdependent pathway recently described by Dower et al. [26] remains to be determined.

Since the TREM-1-mediated activation of the oxidative burst synergizes not only with LPS as TLR4 ligand, but also with ligands, that is, for TLR 2 or TLR7/8, as dem- 
onstrated by us previously [13], it seems possible and likely that the signaling events described here for TREM-1 and TLR4 might also be relevant for other TLRs. Further work is needed to assess whether our results obtained for the activation of signaling pathways of TREM- 1 and TLR4 can be also extended to other TLR ligands. In our studies, we focused on the oxidative burst as a functional read out to account for the immediacy of the molecular effects observed leading to a model of TREM-1 and TLR4 induced signaling cascades as depicted in the proposed model in figure 6 . The use of primary terminally differentiated PMN makes us confident that the signaling events observed are relevant and not skewed by abnormal signals that might occur using PMN-like cell lines such as HL60. However, despite using carefully titrated inhibitor doses with no impairment of the phorbol ester-induced oxidative burst in our pharmacological approach we ultimately cannot exclude nonspecific effects of the inhibitors. Since PMN constitutively undergo apoptosis starting 6-8 $\mathrm{h}$ after onset of in vitro culture [42], it is impossible to use knockdown strategies, for example by siRNA, to validate our findings. Furthermore, it is likely that the signaling events leading to the synergy of TREM1 and TLR4 might follow different pathways in other cell types, such as monocytes, macrophages or most recently described in epithelial cells that also express these recep- tors $[12,15,43,44]$. Further investigations in these cell types are needed for a better understanding of not only the amplifying signals transmitted by TREM-1, but also of the inhibitory functions of DAP12-associated signaling receptors as described for TREM-2 $[15,45,46]$ that we did not observe in our studies.

In conclusion, our present work discloses a major role for p38MAPK- and PI3K-dependent signaling that contribute independently of each other to the amplification of PMN activation via TLR and TREM-1. These results shed a new light on our current understanding of how the innate inflammatory response is regulated and might contribute to the development of future concepts to treat severe inflammatory conditions such as sepsis.

\section{Acknowledgements}

This work was supported by grants from the Deutsche Forschungsgemeinschaft (DFG) to M.P.R. and H.S. (Ra988/2-2/3, Ra988/3-1) and M.S. (Sta984/1-1). The authors express their gratitude to Prof. Dr. Martin Schuler (Department of Internal Medicine, University Hospital Essen, Essen, Germany) and Prof. Dr. Thomas Fischer (3rd Department of Medicine, University Hospital Mainz, Mainz, Germany) for most helpful discussions as well as Anne-Katrin Meinl and Kristian Schütze for excellent technical assistance.

\section{References}

1 Chertov O, Yang D, Howard OM, Oppenheim JJ: Leukocyte granule proteins mobilize innate host defenses and adaptive immune responses. Immunol Rev 2000;177: 68-78.

$\checkmark 2$ Lloyd AR, Oppenheim JJ: Poly's lament: the neglected role of the polymorphonuclear neutrophil in the afferent limb of the immune response. Immunol Today 1992;13: 169-172.

- 3 Sabroe I, Prince LR, Jones EC, Horsburgh MJ, Foster SJ, Vogel SN, Dower SK, Whyte MK: Selective roles for toll-like receptor (TLR) 2 and TLR4 in the regulation of neutrophil activation and life span. J Immunol 2003;170:5268-5275.

4 Kurt-Jones EA, Mandell L, Whitney C, Padgett A, Gosselin K, Newburger PE, Finberg RW: Role of toll-like receptor 2 (TLR2) in neutrophil activation: GM-CSF enhances TLR2 expression and TLR2-mediated interleukin 8 responses in neutrophils. Blood 2002;100:1860-1868.

$\checkmark 5$ Medzhitov R: Toll-like receptors and innate immunity. Nat Rev Immunol 2001;1:135-145.
6 6 Kufer TA, Fritz JH, Philpott DJ: Nacht-lrr proteins (NLRs) in bacterial infection and immunity. Trends Microbiol 2005; 13:381388.

7 Fritz JH, Le Bourhis L, Sellge G, Magalhaes JG, Fsihi H, Kufer TA, Collins C, Viala J, Ferrero RL, Girardin SE, Philpott DJ: Nod1-mediated innate immune recognition of peptidoglycan contributes to the onset of adaptive immunity. Immunity 2007;26:445-459.

8 Tamassia N, Le MV, Calzetti F, Donini M, Gasperini S, Ear T, Cloutier A, Martinez FO, Fabbri M, Locati M, Mantovani A, McDonald PP, Cassatella MA: The MYD88-independent pathway is not mobilized in human neutrophils stimulated via TLR4. J Immunol 2007; 178:7344-7356.

-9 Francois S, El Benna J, Dang PM, Pedruzzi E, Gougerot-Pocidalo MA, Elbim C: Inhibition of neutrophil apoptosis by TLR agonists in whole blood: Involvement of the phosphoinositide 3-kinase/AKT and NF- $\mathrm{KB}$ signaling pathways, leading to increased levels of Mcl-1, A1, and phosphorylated Bad. J Immunol 2005; 174:3633-3642.
10 Yan SR, Al Hertani W, Byers D, Bortolussi R: Lipopolysaccharide-binding protein- and CD14-dependent activation of mitogen-activated protein kinase $\mathrm{p} 38$ by lipopolysaccharide in human neutrophils is associated with priming of respiratory burst. Infect Immun 2002;70:4068-4074.

11 Akira S, Takeda K: Toll-like receptor signalling. Nat Rev Immunol 2004;4:499-511.

12 Bouchon A, Dietrich J, Colonna M: Cutting edge: Inflammatory responses can be triggered by TREM-1, a novel receptor expressed on neutrophils and monocytes. J Immunol 2000;164:4991-4995.

13 Radsak MP, Salih HR, Rammensee HG, Schild $\mathrm{H}$ : Triggering receptor expressed on myeloid cells-1 in neutrophil inflammatory responses: Differential regulation of activation and survival. J Immunol 2004;172: 4956-4963.

14 Haselmayer P, Grosse-Hovest L, von Landenberg P, Schild H, Radsak MP: TREM-1 ligand expression on platelets enhances neutrophil activation. Blood 2007;110:1029-1035. 
15 Bouchon A, Facchetti F, Weigand MA, Colonna M: TREM-1 amplifies inflammation and is a crucial mediator of septic shock. Nature 2001;410:1103-1107.

-16 Gibot S, Buonsanti C, Massin F, Romano M, Kolopp-Sarda MN, Benigni F, Faure GC, Bene MC, Panina-Bordignon P, Passini N, Levy B: Modulation of the triggering receptor expressed on the myeloid cell type 1 pathway in murine septic shock. Infect Immun 2006;74:2823-2830.

17 Gibot S, Massin F, Le Renard P, Bene MC Faure GC, Bollaert PE, Levy B: Surface and soluble triggering receptor expressed on myeloid cells-1: expression patterns in murine sepsis. Crit Care Med 2005;33:1787-1793.

-18 Wong-Baeza I, Gonzalez-Roldan N, FeratOsorio E, Esquivel-Callejas N, Aduna-Vicente R, Arriaga-Pizano L, Astudillo-de la Vega H, Villasis-Keever MA, Torres-Gonzalez R, Estrada-Garcia I, Lopez-Macias C, Isibasi A: Triggering receptor expressed on myeloid cells (TREM-1) is regulated posttranscriptionally and its ligand is present in the sera of some septic patients. Clin Exp Immunol 2006;145:448-455.

19 Gibot S, Massin F: Soluble form of the triggering receptor expressed on myeloid cells 1 : an anti-inflammatory mediator? Intensive Care Med 2006;32:185-187.

20 Mohamadzadeh M, Coberley SS, Olinger GG, Kalina WV, Ruthel G, Fuller CL, Swenson DL, Pratt WD, Kuhns DB, Schmaljohn AL: Activation of triggering receptor expressed on myeloid cells-1 on human neutrophils by marburg and ebola viruses. J Virol 2006;80:7235-7244.

-21 Klesney-Tait J, Turnbull IR, Colonna M: The TREM receptor family and signal integration. Nat Immunol 2006;7:1266-1273.

-22 Tessarz AS, Weiler S, Zanzinger K, Angelisova $P$, Horejsi V, Cerwenka A: Non-T cell activation linker (NTAL) negatively regulates TREM-1/DAP12-induced inflammatory cytokine production in myeloid cells. J Immunol 2007;178:1991-1999.

-23 Netea MG, Azam T, Ferwerda G, Girardin $\mathrm{SE}$, Kim SH, Dinarello CA: Triggering receptor expressed on myeloid cells-1 (TREM-1) amplifies the signals induced by the NACHTLRR (NLR) pattern recognition receptors. J Leukoc Biol 2006;80:1454-1461.

-24 El Mezayen R, El Gazzar M, Seeds MC, McCall CE, Dreskin SC, Nicolls MR: Endogenous signals released from necrotic cells augment inflammatory responses to bacterial endotoxin. Immunol Lett 2007;111:36-44.
25 Fortin CF, Lesur O, Fulop T Jr: Effects of trem-1 activation in human neutrophils: activation of signaling pathways, recruitment into lipid rafts and association with TLR4. Int Immunol 2007;19:41-50.

26 Dower K, Ellis DK, Saraf K, Jelinsky SA, Lin LL: Innate immune responses to trem-1 activation: overlap, divergence, and positive and negative cross-talk with bacterial lipopolysaccharide. J Immunol 2008;180:35203534.

27 Radsak MP, Hilf N, Singh-Jasuja H, Braedel S, Brossart P, Rammensee HG, Schild H: The heat shock protein Gp96 binds to human neutrophils and monocytes and stimulates effector functions. Blood 2003;101:2810 2815.

28 Turnbull IR, Colonna M: Activating and inhibitory functions of DAP12. Nat Rev Immunol 2007;7:155-161.

29 Tessarz AS, Cerwenka A: The TREM-1/ DAP12 pathway. Immunol Lett 2008;116: 111-116.

30 Young PR, McLaughlin MM, Kumar S, Kassis S, Doyle ML, McNulty D, Gallagher TF, Fisher S, McDonnell PC, Carr SA, Huddleston MJ, Seibel G, Porter TG, Livi GP, Adams JL, Lee JC: Pyridinyl imidazole inhibitors of p38 mitogen-activated protein kinase bind in the ATP site. J Biol Chem 1997;272: 12116-12121.

31 Dusi S, Donini M, Della BV, Rossi F: Tyrosine phosphorylation of phospholipase $c-\gamma 2$ is involved in the activation of phosphoinositide hydrolysis by FC receptors in human neutrophils. Biochem Biophys Res Commun 1994;201:1100-1108.

32 Majumdar S, Kane LH, Rossi MW, Volpp BD, Nauseef WM, Korchak HM: Protein kinase $c$ isotypes and signal-transduction in human neutrophils: Selective substrate specificity of calcium-dependent $\beta$-PKC and novel calcium-independent NPKC. Biochim Biophys Acta 1993;1176:276-286.

33 Rodeberg DA, Babcock GF: Role of calcium during lipopolysaccharide stimulation of neutrophils. Infect Immun 1996;64:28122816.

34 Yamamori T, Inanami O, Nagahata H, Cui Y, Kuwabara M: Roles of p38 MAPK, PKC and $\mathrm{PI} 3-\mathrm{K}$ in the signaling pathways of NADPH oxidase activation and phagocytosis in bovine polymorphonuclear leukocytes. FEBS Lett 2000;467:253-258.

35 Hirsch E, Katanaev VL, Garlanda C, Azzolino O, Pirola L, Silengo L, Sozzani S, Mantovani A, Altruda F, Wymann MP: Central role for g protein-coupled phosphoinositide 3 -kinase $\gamma$ in inflammation. Science 2000; 287:1049-1053.
36 Ivashkiv LB: A signal-switch hypothesis for cross-regulation of cytokine and TLR signalling pathways. Nat Rev Immunol 2009;8: $816-822$.

-37 Toshchakov VY, Vogel SN: Cell-penetrating TIR BB loop decoy peptides a novel class of TLR signaling inhibitors and a tool to study topology of TIR-TIR interactions. Expert Opin Biol Ther 2007; 7:1035-1050.

38 Vabulas RM, Braedel S, Hilf N, Singh-Jasuja H, Herter S, Ahmad-Nejad P, Kirschning CJ, Da Costa C, Rammensee HG, Wagner H, Schild H: The endoplasmic reticulum-resident heat shock protein Gp96 activates dendritic cells via the Toll-like receptor $2 / 4$ pathway. J Biol Chem 2002;277:20847-20853.

>39 Chang L, Karin M: Mammalian MAP kinase signalling cascades. Nature 2001;410:37-40.

40 O’Neill LA, Bowie AG: The family of five: TIR-domain-containing adaptors in Tolllike receptor signalling. Nat Rev Immunol 2007;7:353-364.

-41 Brown GE, Stewart MQ, Bissonnette SA, Elia AE, Wilker E, Yaffe MB: Distinct ligand-dependent roles for $38 \mathrm{MAPK}$ in priming and activation of the neutrophil NADPH oxidase. J Biol Chem 2004;279:27059-27068.

42 Luo HR, Loison F: Constitutive neutrophil apoptosis: mechanisms and regulation. Am J Hematol 2008;83:288-295.

-43 Schmausser B, Endrich S, Beier D, Moran AP, Burek CJ, Rosenwald A, Rieckmann P, Muller-Hermelink HK, Eck M: Triggering receptor expressed on myeloid cells-1 (TREM-1) expression on gastric epithelium: implication for a role of TREM-1 in Helicobacter pylori infection. Clin Exp Immunol 2008;152:88-94.

44 Schenk M, Bouchon A, Birrer S, Colonna M, Mueller C: Macrophages expressing triggering receptor expressed on myeloid cells-1 are underrepresented in the human intestine. J Immunol 2005; 174:517-524.

45 Hamerman JA, Jarjoura JR, Humphrey MB, Nakamura MC, Seaman WE, Lanier LL: Cutting edge: inhibition of TLR and FCR responses in macrophages by triggering receptor expressed on myeloid cells (TREM)-2 and DAP12. J Immunol 2006;177:20512055.

46 Hamerman JA, Tchao NK, Lowell CA, Lanier LL: Enhanced toll-like receptor responses in the absence of signaling adaptor DAP12. Nat Immunol 2005;6:579-586. 